October 28,2018

\title{
Detecting Casimir Forces through a Tunneling Electromechanical Transducer
}

\author{
Roberto Onofrio ${ }^{1,2}$ and Giovanni Carugno ${ }^{2}$ \\ in 1 Dipartimento di Fisica "G. Galilei", Università di Padova, Via Marzolo 8, Padova, Italy 35131 \\ ${ }^{2}$ Istituto Nazionale di Fisica Nucleare, Sezione di Padova, Italy 35131
}

(October 28, 2018)

\begin{abstract}
We propose the use of a tunneling electromechanical transducer to dinamically detect Casimir forces between two conducting surfaces. The maximum distance for which Casimir forces should be detectable with our method is around $1 \mu \mathrm{m}$, while the lower limit is given by the ability to approach the surfaces. This technique should permit to study gravitational forces on the same range of distances, as well as the vacuum friction provided that very low dissipation mechanical resonators are used.
\end{abstract}

03.70.+k, 06.70.Mx, 73.40.Gk

Typeset using REVTEX 
One of the most astonishing and less understood consequences of the existence of quantum vacuum is the possibility to observe forces between macroscopic neutral bodies, as outlined in a pioneering paper by Casimir [1]. Casimir forces are of purely relativistic origin and this is manifested by their retarded nature, thus vanishing at very small distances. The force per unit area between two conducting, but neutral, indefinite and parallel plates has been calculated as

$$
P_{C}=\frac{K_{C}}{d^{4}}
$$

where $d$ is the distance between the two plates, assumed greater than the penetration lenght in the conductors $\simeq 100 \mathrm{~nm}$, and $K_{C}=\pi h c / 480=1.3 \cdot 10^{-27} \mathrm{Nm}^{2}$ is a purely kinematic constant depending upon the speed of light $c$ and the Planck constant $h$. Until now few experiments have been performed to verify this simple law. The submicron region has been explored in the range $0.5 \div 2 \mu \mathrm{m}$ [2]. Measurements have also been reported in the range $50 \div 130 \mathrm{~nm}$ [5] while a proposal aimed at observing Casimir forces in the cm range using optical techniques has been recently discussed in [6]. In this Letter we propose a detection technique for macroscopic forces and we report on preliminary results concerning the calibration of a prototype for the observation of Casimir forces by means of a tunneling electromechanical transducer. Our estimates suggest that the Casimir force should be detectable at least in a range of two decades below $10 \mu \mathrm{m}$.

The detection scheme is schematically shown in Fig. 1. The two parallel conducting surfaces are made of a cantilever beam with a mass of circular shape at its extreme, rigidly clamped to the other end on a base in such a way to oscillate, and a disk rigidly connected to a piezoelectric stack. The distance between these two surfaces can be changed both by driving the mechanical oscillator at its proper frequency $\nu_{r}$ through a piezoelectric gauge and by driving the disk at a fixed frequency $\nu_{p}$ through the piezoelectric stack. For practical reasons the vibration frequency of the piezoelectric stack is smaller than the one of the mechanical resonator. On the other side of the resonator a tunneling electromechanical transducer allows to measure the amplitude of its oscillations. When the disk approaches 
the resonator the attractive Casimir force should appear and a static displacement should result in a decrease of the tunneling current detected on the other side of the resonator. However this measurement is affected by all the usual drawbacks of a static detection and this is true also for a very low-frequency detection scheme due the presence of $1 / f$-like noise of mechanical and electrical nature. The heterodyne detection, as discussed in detail in [7] is unaffected by this problem: let us suppose that both the resonator and the disk are sinusoidally driven at the angular frequencies $\omega_{r}=2 \pi \nu_{r}$ and $\omega_{p}=2 \pi \nu_{p}$ and the amplitudes of the corresponding oscillations are respectively $x_{r}$ and $x_{p}$. Thus the gap between the two plates will become time-dependent as

$$
d(t)=d_{0}+x_{r} \cos \left(\omega_{r} t\right)+x_{p} \cos \left(\omega_{p} t\right)
$$

The Casimir force will be therefore modulated and, by supposing that the infinite plane is a valid approximation for a plate of finite surfaces, its expression will be written as

$$
F_{C}(t) \simeq \frac{K_{C} S}{d_{0}^{4}\left[1+\left(x_{r} / d_{0}\right) \cos \omega_{r} t+\left(x_{p} / d_{0}\right) \cos \omega_{p} t\right]^{4}}
$$

If we suppose a weak modulation regime in (2), i.e. $x_{r}, x_{p}<<d_{0}$, we obtain, up to the third order in $x_{r} / d_{0}, x_{p} / d_{0}$

$$
F_{C}(t)=K_{C} \frac{S}{d_{0}^{4}}[1+g(t)]
$$

where $g(t)$ has Fourier components shown in Tab. I. These Fourier components of the Casimir force give rise to Fourier components for the motion of the mechanical resonator at the same frequencies, which are finally detected by the tunneling tip. The relationship between force and displacement is given by the mechanical transfer function of the resonator. When the disk is put apart enough from the resonator only the component driven at mechanical resonance $\omega_{r}$ survives. The other linear component at $\omega_{p}$ can be also exploited to get information on the presence of forces but an accurate shielding from the vibrations induced in the resonator by the PZT stack is required. Moreover the presence of $1 / \mathrm{f}$ noise in the tunneling current limits the possibility of exploiting this low-frequency harmonic as well 
as the other one at $2 \omega_{p}$. The existence of a very small modulation parameter $x_{r}$, being for practical reasons $x_{r}<<x_{p}$, provides rationale for ruling out the Fourier component at $2 \omega_{r}$, which should be also affected by the non-linear relationship between tunneling current and tip-sample gap. The lateral bands around the resonant frequency seem the best candidates to obtain information on the Casimir force, being less plagued by seismic noise or electric $1 / \mathrm{f}$ noise in the tunneling transducer. On the other hand these two components are suppressed by a factor $10 x_{r} x_{p} / d_{0}^{2}$ with respect to the static case.

The considerations made above are quite conservative and assume for instance that no accurate acoustic insulation has been provided to prevent acoustic pick-up at $\omega_{p}$ due to the PZT stack. Less pessimistic considerations can only enforce our estimates also by using a redundancy among the informations present at the different Fourier components.

This detection scheme has been tested through a first calibration by biasing at constant voltage the gap formed between the resonator and the disk. The apparatus used is schematically shown in Fig.2. The tunneling electromechanical transducer is similar to the one already described in [8] and its sensitivity is of the order of $10^{-11} \mathrm{~m}$ at a frequency of $60 \mathrm{KHz}$. Its main advantages with respect to other types of transducers are the possibility of monitoring small masses with enough sensitivity and the absence of problems related to the parallelism, the probe being point-like. Unlike in ref. [8] the approach of the tip to the surface of the resonator is obtained through an inchworm motor [9]. The resonator is made out of a single piece of steel machined through electroerosion and its vibrating mass is a cylinder with diameter of $6 \mathrm{~mm}$ and a thickness of $0.2 \mathrm{~mm}$. The lenght of the cantilever beam, with a rectangular section of $0.1 \mathrm{~mm}$ times $0.2 \mathrm{~mm}$, is equal to $4 \mathrm{~mm}$. A schematic view of the resonator is shown in the upper-left part of Fig.3. In the same figure the mechanical transfer function, obtained by the FFT of the tunneling current under white noise mechanical excitation, is shown. A frequency for the first flexural mode of the cylinder $\nu_{r}=19 \mathrm{KHz}$ and a mechanical quality factor of $10^{2}$ are measured. The piezoelectric stack has a distance modulation per unit of voltage equal to $0.2 \mu \mathrm{m} / \mathrm{V}$ with a high-frequency cutoff around $5 \mathrm{KHz}$. It is connected to a flat surface of Aluminum, $10 \mathrm{~mm}$ diameter, $4 \mathrm{~mm}$ 
thick. The apparatus is put on a four cantilever beam suspension similar to the ones used for suspension of gravitational wave detectors [10]. The disk is biased at constant voltage $\mathrm{V}$ and the resulting force acting on the resonator is, up to second order,

$$
F_{V}(t)=\frac{C V^{2}}{2 d(t)} \simeq \frac{\epsilon_{0} S}{2}\left(\frac{V}{d_{0}}\right)^{2}\left(1-\frac{x_{r}}{d_{0}} \cos \omega_{r} t-\frac{x_{p}}{d_{0}} \cos \omega_{p} t\right)^{2},
$$

which contains also terms at the lateral bands $\omega_{r} \pm \omega_{p}$ of amplitude equal to

$$
F_{V}\left(\omega_{r} \pm \omega_{p}\right)=\frac{\epsilon_{0} S}{2}\left(\frac{V}{d_{0}}\right)^{2} \frac{x_{r}}{d_{0}} \frac{x_{p}}{d_{0}}
$$

This has to be compared with the component of the Casimir force at the lateral bands as deduced from Eqn.4 and Table I:

$$
F_{C}\left(\omega_{r} \pm \omega_{p}\right)=10 K_{C} \frac{S}{d_{0}^{4}} \frac{x_{p}}{d_{0}} \frac{x_{r}}{d_{0}}
$$

To get an estimate of the sensitivity it is useful to introduce an equivalent voltage $V_{e q}$ such that for a given distance $d_{0}$ a bias equal to $V_{e q}$ simulates the Casimir force. This equivalent voltage is independent of the modulation parameters and, by equating Eqns. (6) and (4)), we get

$$
V_{e q}=\left(\frac{20 K_{C}}{\epsilon_{0}}\right)^{1 / 2} \frac{1}{d_{0}}=\frac{5.42 \cdot 10^{-8}}{d_{0}}
$$

which means, for instance, that the Casimir force at distance $d_{0}=10^{-6} \mathrm{~m}$ can be simulated by a bias voltage $V_{e q} \simeq 50 \mathrm{mV}$. Lateral bands due to the electric field are observed in Fig. 4b,c which show their appearance only when the bias voltage and the driving voltage for the piezoelectric stack are both present. By comparison we also report in Fig.4a the spectrum observed in absence of the bias voltage. The two cases of Fig. 4b,c are relative to $V=150 \mathrm{~V}$ and $V=300 \mathrm{~V}$ respectively. In the latter case the lateral peaks are above the noise by a factor 6. From this we deduce that a signal to noise ratio $\mathrm{SNR}=1$ corresponds to $V_{e q}^{S N R=1} \simeq 70 \mathrm{~V}$. This has to be compared with the equivalent voltage of the Casimir force at $d_{0}=10^{-6} \mathrm{~m}$ which is three orders of magnitude smaller. Many improvements can be made on a dedicated prototype to increase the sensitivity by three orders of magnitude. Resonators with larger 
mechanical quality factors and operating in vacuum can be used. The wide-band noise can be reduced by optimizing the electronic chain. Finally, phase sensitive detection techniques can be exploited. The first tests show that more than a factor 10 in the sensitivity is gained by using the vector averaging mode of the FFT spectrum analyzer. The ultimate limit on the reduction of the noise uncorrelated with the signal is determined in this last case by the stability of the tunneling current during the integration time, which we observe to be constant at least on a scale of tens of minutes.

The lower limit of the distance at which Casimir forces are measurable is given by the ability to control the distance between the two plates. It is possible to put the previous apparatus inside an electron microscope and to use piezoelectric quadrant tubes to control the parallelism. In this way uniform gaps of the order of $100 \mathrm{~nm}$ or less can be obtained, allowing for a quantitative test within two orders of magnitude of the distance. Flat surfaces on the scale of less than 100nm can be obtained exploiting polishing techniques already used in optical devices. Preliminary tests have been performed on microresonators and micrometers to check the feasibility of a monitoring of surfaces gaps in the 100nm range inside an electron microscope.

Besides the study of the Casimir force we believe this detection technique could also be exploited for the measurement of other forces spatially dependent on the same range of distances, such as gravitational forces. In this case particular configurations should be used for the geometry of the test masses to allow very small distances between the center of mass of the bodies. The dependence upon the distance can be also determined by an accurate measurement of the Fourier components at a given distance. By repeating the previous considerations for a generic force of the type

$$
F_{n}=\frac{K}{d^{n}}
$$

we get a dynamical force

$$
F_{n}(t)=\frac{K}{d_{0}^{n}}\left[1+g_{n}(t)\right]
$$


where $g_{n}(t)$ has Fourier components, up to the second order, shown in Tab. II. The various components depend upon $\mathrm{n}$, which in turn can be measured by determining for instance the ratio

$$
G_{n}\left(\omega_{p}\right) / G_{n}\left(\omega_{r} \pm \omega_{p}\right)=\frac{2}{n+1} \frac{d_{0}}{x_{r}}
$$

where $d_{0} / x_{r}$ can be determined using a simultaneous calibration with an electric field. To observe the Fourier component at $\omega_{p}$ an accurate shielding from the vibration induced by the PZT stack is mandatory. For n of physical interest (gravitational forces, Casimir forces, i.e. $\mathrm{n}=2,4$ ) the differences in the values are above 20 per cent and should be detectable, opening also the possibility to study gravity on $\mu \mathrm{m}$ distances with geometries of the bodies such that the center of mass can be spaced by such order of magnitude. The static gravitational force between the masses used in our prototype is $F_{G} \simeq 6 \cdot 10^{-7} \mathrm{~N}$ supposing that the centers of mass are spaced by $1 \mu \mathrm{m}$, i.e. one order of magnitude larger that the corresponding Casimir force.

The proposed detection method is also valid far from the mechanical resonance. However the use of a resonance condition is welcome because of the gain, equal to the mechanical quality factor, with respect to a non-resonant configuration. Use of microresonators with very high mechanical quality factors could also allow for an alternative detection scheme based upon the measurement of the resonant frequency shift induced by the Casimir force. Furthermore, a resonance condition has been recently suggested [11], 12 to detect the dissipative part of the Casimir force as predicted in [13]. Although this requires very high mechanical quality factors, the improvements made in the technology of microresonators [14], [15] allow to hope that in a close future the dissipative nature of the quantum vacuum could be detected and studied.

\section{ACKNOWLEDGMENTS}

We acknowledge V. B. Braginsky for fruitful discussions and A. Bettini, M. Cerdonio, E. Conti, F. Illuminati and G. Ruoso for a critical reading of the manuscript. 


\section{REFERENCES}

[1] H. B. Casimir, Proc. K. Ned. Akad. Wet. 51, 793 (1948).

[2] M. J. Sparnay, Nature 180, 334 (1957).

[3] M. J. Sparnay, Physica 24, 751 (1958).

[4] A. Sarlemijn and M. J. Sparnaay, Physics in the Making (North-Holland, Amsterdam, 1989), pp. 235-246; for a review including also work done with Rydberg spectroscopy see Long-range Casimir forces, theory and recent experiments on atomic systems, F. S. Levin and D. A. Miche editors (Plenum Press, New York, 1993).

[5] J. N. Israelachvili and D. Tabor, Proc. R. Soc. London, Scr. A 331, 19 (1972).

[6] E. Jacopini, Phys. Rev. A 48, 129 (1993).

[7] S. Hunklinger, H. Geisselmann, and W. Arnold, Rev. Sci. Instr. 43, 584 (1972); for a nice application to the study of van der Waals forces in the $0.2 \div 1 \mu m$ range see also W. Arnold, S. Hunklinger, and K. Dransfeld, Phys. Rev. B 19, 6049 (1979).

[8] G. Carugno, E. Conti and R. Onofrio, Europhys. Lett. 24, 713 (1993).

[9] Burleigh Instruments, Inc., Burleigh Park, Fishers, NY 14453.

[10] E. Coccia, Rev. Sci. Instrum. 53, 148 (1982).

[11] V. B. Braginsky and F. Ya. Khalili, Phys. Lett. A 161, 197 (1991).

[12] V. B. Braginsky and F. Ya. Khalili, Phys. Lett. A 167, 1 (1992).

[13] L. S. Levitov, Europhys. Lett. 8, 499 (1989).

[14] G. Kaminsky, J. Vac. Sci. Techn. B 3, 1015 (1985).

[15] R. A. Buser and N. F. De Rooij, Sensors and Actuators, A 21-23, 323 (1990). 


\section{TABLES}

\begin{tabular}{|c|c|}
\hline \hline$\omega$ & $G(\omega)$ \\
\hline$\omega_{p}$ & \\
$2 \omega_{p}$ & $4 x_{p} / d_{0}$ \\
$\omega_{r}-\omega_{p}$ & $5\left(x_{p} / d_{0}\right)^{2}$ \\
$\omega_{r}$ & $10 x_{p} x_{r} / d_{0}^{2}$ \\
$\omega_{r}+\omega_{p}$ & $4 x_{r} / d_{0}$ \\
$2 \omega_{r}$ & $10 x_{p} x_{r} / d_{0}^{2}$ \\
\hline \hline
\end{tabular}

TABLE I. Fourier components of the dynamical term $g(t)$

\begin{tabular}{|c|c|}
\hline \hline$\omega$ & $G_{n}(\omega)$ \\
\hline$\omega_{p}$ & $n x_{p} / d_{0}$ \\
$2 \omega_{p}$ & $\frac{n(n+1)}{4}\left(x_{p} / d_{0}\right)^{2}$ \\
$\omega_{r}-\omega_{p}$ & $\frac{n(n+1)}{2} x_{p} x_{r} / d_{0}^{2}$ \\
$\omega_{r}$ & $n x_{r} / d_{0}$ \\
$\omega_{r}+\omega_{p}$ & $\frac{n(n+1)}{2} x_{p} x_{r} / d_{0}^{2}$ \\
$2 \omega_{r}$ & $\frac{n(n+1)}{4}\left(x_{r} / d_{0}\right)^{2}$ \\
\hline \hline
\end{tabular}

TABLE II. Fourier components of the dynamical term $g_{n}(t)$ 


\section{FIGURES}

FIG. 1. Dynamical detection scheme for small forces acting on a micromechanical resonator.

FIG. 2. Scheme of the apparatus used in the calibration.

FIG. 3. FFT spectrum of the tunneling current under white-noise excitation of the resonator whose shape is drawn in the upper-left corner. The curve is proportional to the transfer function of the mechanical oscillator, and allows to measure resonant frequency and mechanical quality factor.

FIG. 4. FFT Spectrum of the tunneling current in presence of a mechanical modulation of the resonator without (a) and with (b,c) a mechanical modulation of the disk under biasing at constant voltage respectively at $V=150 \mathrm{~V}$ and $V=300 \mathrm{~V}$. Lateral bands at $\omega_{r} \pm \omega_{p}$ are observable with amplitude depending both upon the driving voltage of the piezoelectric stack and the bias voltage. The lateral bands in b) are about $12 \mathrm{~dB}$ below the ones in c) which corresponds to a quadratic scaling for the signal versus the bias voltage. In the case c) we have $\nu_{r}=19.55 \mathrm{KHz}, \nu_{p}=1 \mathrm{KHz}$, $V=300 \mathrm{~V}, d_{0}=10^{-4} \mathrm{~m}, x_{r} \simeq 10^{-9} \mathrm{~m}, x_{p}=2 \cdot 10^{-7} \mathrm{~m}, S=2.83 \cdot 10^{-5} \mathrm{~m}^{2}$ which implies a Fourier component of the force $F_{V}\left(\omega_{r} \pm \omega_{p}\right) \simeq 2 \cdot 10^{-11} \mathrm{~N}$. 
This figure "fig1-1.png" is available in "png" format from: http://arxiv.org/ps/quant-ph/9503001v1 
This figure "fig2-1.png" is available in "png" format from: http://arxiv.org/ps/quant-ph/9503001v1 
This figure "fig1-2.png" is available in "png" format from: http://arxiv.org/ps/quant-ph/9503001v1 
This figure "fig1-3.png" is available in "png" format from: http://arxiv.org/ps/quant-ph/9503001v1 